\title{
ESPACIOS EDUCATIVOS ABIERTOS: HACIA UNA ANIMACIÓN COMUNITARIA INTEGRADORA Y TRANSFORMADORA
}

\author{
Open educative spaces: towards an communitary \\ animation integrative and changeable activity
}

\section{Sindo FROUFE QUINTAS}

Universidad de Salamanca. Facultad de Ciencias Sociales. Departamento de Teoría e Historia de la Educación. Campus "Miguel de Unamuno". Edificio F.E.S. 37007 Salamanca. Correo-e: sfq@usal.es

Fecha de aceptación definitiva: marzo de 2001

BIBLID [(1130-3743) 12, 2000, 107-128]

\section{RESUMEN}

El desarrollo de las comunidades locales y la animación comunitaria se están convirtiendo en la actualidad en una de las metodologías para la mejora del tejido social próximo a los problemas concretos de la gente. Esbozamos cómo la animación comunitaria realizada en/con/para la comunidad es el ámbito para la producción de cambios sociales que redunden en beneficio de los colectivos marginados. Realizamos un estudio previo acerca de la concepción de la comunidad actual y de sus nuevas realidades y ofrecemos una serie de conclusiones como propuestas abiertas para el debate.

Descriptores: animación comunitaria, comunidad, participación, compromiso social, transformación social, valores. 
SUMMARY

The development of the local communities and the communitary animation are converting nowadays as one of the methodologies for the improvement of the social weave near to the concrete problems of the people. We sketch how the communitary animation made in/with/for the community is the propitious field for the production of social changes that benefit to the outcast groups. We realice a previous study about the conception of the current community and about its new realities and we offer a set of conclusions as open proposals for the debate.

Key words: communitary animation, community, participation, social commitment, social transformation, values.

\section{CONTEXTUALIZACiÓN}

El ser humano se abre al mundo en contacto real con distintos espacios o contextos (Schultz, 1993; Robottom, 1987) que influyen en su maduración y desarrollo personal. Estos espacios educativos abiertos, llámese familia, grupos primarios, vecindad, comunidad inmediata, escuela, territorio, hábitat, ambiente, etc., deben organizarse de forma consciente, reflexiva e integradora para que sus funciones y actuaciones se dirijan hacia un mismo objetivo y su finalidad sea eminentemente educativa. Los contextos acaban convirtiéndose en espacios y tiempos sociales (también educativos) y sus diferentes componentes (simbólicos y materiales) coinciden en otorgarle el significado de soporte principal para el desarrollo de la vida, en las variadas formas en las que ésta se manifiesta. De ahí nace su importancia para explicar el progreso científico que no debe limitarse a una mera labor de reconstrucción racional, sino que necesita incorporar factores histórico-sociales en sus explicaciones (Kuhn, 1985; Gurwitsch, 1990; Tynieniecka, 1996). "Los mayores problemas educativos surgen cuando se producen incongruencias estructurales entre los distintos órdenes comunitarios" ${ }^{1}$. Vivir en comunidad es una exigencia de la socialización humana y de la educación cósmica (Nassif, 1980).

Proponemos - nuestra experiencia profesional así nos lo confirma- a la animación comunitaria como nueva forma de acción socioeducativa en las comunidades locales o municipios. Significa interpretar el territorio como un espacio de espacios abiertos al aprendizaje y a la socialización. La identidad de grupo (Dunleavy, 1991), todo aquello que es común a muchas personas y que les une en la forma de interpretar y vivir el mundo, se confirma en la actualidad como uno de los modelos/métodos más provechosos y dinámicos para la solución de conflictos y problemas sociales dentro del amplio panorama de las Ciencias de la Educación.

1. Rodríguez Neira, T. (1996): “Aprendizajes tácitos: comunidades, grupos y casas". Aula Abierta, 68, 12. 
De ahí el interés que merece en el campo de las dinámicas de grupo activas todo aquello que se refiera a grupo, asociación, vecindad, cooperativismo, comunidad local, desarrollo local, municipio, etc. Actualmente podemos afirmar que el ámbito de lo local, de lo cercano, de lo próximo e inmediato a las personas y colectivos sociales es el ecoterritorio más adecuado y propicio para una acción educativa comunitaria desde el amplio y complejo enfoque de la Teoría de la Educación. El desarrollo de la comunidad se percibe hoy como una forma de sensibilización y gestión educativa en favor de las personas para que participen en la solución de sus propios problemas y en la búsqueda de una mejor calidad de vida. Todos estos contextos abiertos y comunitarios "son tentativas en las que se reflejan deseos, esperanzas y tensiones del hombre situadas en el contexto de sus condiciones sociales" (Popkewitz, 1988: 39). Estamos de acuerdo con las palabras de Rodríguez Neira $^{2}$ :

"Urge tomar de nuevo las comunidades inmediatas, los grupos primarios y los domicilios para ver las propiedades que los definen y las respuestas que desde ellos se pueden ofrecer a la existencia. Una intervención educativa sensata pasa por la comprensión de estos ámbitos».

Las potencialidades del desarrollo local se fotografían en las siguientes razones: es el ámbito en el que se actúa para mejorar las condiciones de vida de la gente, principalmente de los sectores populares y socialmente desfavorecidos; fortalece a los gobiernos locales y a todas sus secciones; amplía el poder de las organizaciones comunitarias y de las redes sociales, constituyendo todo ello la mejor fórmula de consolidación de la democracia basada en la participación activa de todos los sectores de la sociedad. Bassand $(1992)^{3}$ escribe:

"El desarrollo local se revela como un proyecto de sociedad que estimula el diálogo entre actores de lo más variado de las sociedades contemporáneas. El análisis de lo local se convierte en uno de los más fructíferos y ricos de las ciencias sociales".

El desarrollo local como ámbito privilegiado de las acciones microsociales se convierte en un medio de fortalecimiento de los gobiernos municipales, así como la medicina más adecuada para mejorar las condiciones de vida de los sectores populares. El desarrollo local pretende reconstruir las redes de solidaridad, dar apoyo a la organización colectiva de los habitantes y promover nuevos líderes que representen a los excluidos socialmente. Hasta ahora los modelos macrosociales, defendidos a partir de 1963 por las Naciones Unidas, han estado muy lejos de los problemas reales y diarios de la gente. El enfoque más extendido por estas calendas era que el desarrollo de la comunidad era una manera de contribuir al desarrollo

2. Rodríguez Nerra, T. (1999): “Lo privado. Aprendizajes tácitos”. Teoria de la Educación, 11, 87

3. BASSAND, M. (1992): Cultura y regiones de Europa. Oikos-Tau, Barcelona, p. 117. 
nacional. Nada más lejos de la realidad. El desarrollo de la comunidad se interpreta en la actualidad como una forma de sensibilización y motivación de la gente para que participen en la solución de todos aquellos problemas propios que pueden resolver con su actuación porque están a su alcance y en su mundo particular. Sin embargo, la preocupación creciente de lo local no debe eclipsar la visión de lo global. La posible dicotomía debemos reforzarla mediante un nuevo planteamiento armónico: actuar localmente, pero pensando globalmente. Si nos encerramos en nuestro mundo local y privado como lo único válido y viable, perdemos la perspectiva de los cambios globales que se dan continuamente en nuestra sociedad en todos los sectores de la vida. Y más en esta sociedad de las tecnologías de la información donde todo avanza a ritmos jamás pensados ni conocidos.

Las metodologías comunitarias que se apoyan en los elementos ecológicos y contextuales donde vive la persona que necesita la ayuda de los demás para salir de sus conductas inadaptadas o de sus ambientes empobrecidos culturalmente, es la mejor estrategia pedagógico-educativa para que esa persona o esos colectivos marginados cambien sus formas de comportamiento, siempre que sea la comunidad la que potencie su autoestima y les sugiera el aprendizaje de las reglas o habilidades sociales de convivencia humana.

Las redes sociales y ecológicas que diseñan el modelo comunitario enfatizan en sus principios la necesidad de potenciar las perspectivas preventivas en todas las esferas de su actuación, antes que las estrictamente reparadoras. Los modelos comunitarios actúan en el ambiente natural de la persona, adoptando un planteamiento ecológico en sus propuestas, desde donde se potencian las relaciones entre las personas y su ambiente físico-social. El contexto sociocultural se convierte en un territorio educativo. Promueven los cambios institucionales con la finalidad de que las instituciones sociales sean receptivas a las necesidades de todo tipo de la población, objeto primario, prioritario y directo de la intervención. El individuo/persona y la comunidad deben actuar como un todo integrado, compacto e interactivo.

El desarrollo de la comunidad exige la participación y la movilización de los recursos humanos para poder llevar a cabo los programas comunitarios donde las personas o los colectivos sociales se pongan en estado de movilización porque lo que vamos a realizar/ejecutar concierne directamente a sus intereses. La participación comunitaria promueve la conciencia individual y colectiva hacia un compromiso porque siempre se espera que se consiga algo para nosotros. De lo contrario, las gentes no participarían. Se quedarían encerradas en su mundo familiar, desconfiando de las buenas intenciones y aportaciones de los demás.

La Administración local como la institución más próxima a los ciudadanos/as es el interlocutor propicio que mejor puede captar las necesidades, los problemas, las inquietudes y las exigencias de las personas. Sin duda el municipio es el espacio local en el que se da la expresión de los derechos para la vida, cuya satisfacción se hace de una forma más colectiva e interdependiente. Como escribe Colom (1985: 37): 
"El municipio ha sido y es el gran escaparate de la generación cultural popular, encauzador de la participación ciudadana y también, en la gran mayoría de los casos, el contexto en donde se evidencia más claramente la aportación de bienes sociales que desde la Administración se pretende hacer llegar a los ciudadanos".

Es obligado insertar en las áreas de gestión municipal medidas de acción e intervención de tipo positivo que generen una cultura de la igualdad y un cambio en la mentalidad de las personas a favor de una mejora de nivel y de calidad de vida de todos los ciudadanos. Esta conciencia colectiva de lucha por los bienes comunes servirá de antesala a los posibles programas de intervención social en cada municipio, barrio o pueblo. Sólo así se unirán las fuerzas disgregadas de los distintos agentes sociales en defensa de la cultura popular y de todas sus manifestaciones.

Las administraciones locales por su proximidad a los ciudadanos/as están en inmejorables condiciones para promover, impulsar, rescatar y elaborar proyectos educativos y culturales dirigidos a cualquier colectivo social asentado en su territorio. El espacio local permite la reflexión acerca de las formas culturales de la vida cotidiana y el reconocimiento de todos aquellos valores que refuerzan la interacción social. Sin embargo, no podemos obviar que:

"Las instituciones que organizan la política cultural cuentan con sus propias características y pautas de comportamiento, y sus instrumentos políticos determinan un modo decisivo de resultado" (Street, 2000: 106).

\section{El REDESCUBRIMIENTO DE LA COMUNIDAD MODERNA}

Se impone a principios del siglo xxI una revisión en profundidad del concepto de comunidad, ya que el redescubrimiento de la comunidad moderna obliga a la creación de mundos privados, mediatizados por la búsqueda de soluciones personales a casi todos los problemas que acucian a la familia o a su mundo más inmediato. La comunidad "es el lugar de la convivencia y es el medio de circulación de los instrumentos iniciales de la posición del hombre ante el mundo y su interpretación" (Rodríguez Neira, 1999: 88). Actualmente nos vemos obligados a realizar una revisión en extensión del concepto de la comunidad tradicional. Desde el siglo pasado distintos pensadores (Caplan, 1964; Etzioni, 1968; Tönnies, 1979; Wallerstein, 1982; Deligny, 1982; Quintana, 1984; Roth, 1986; Ware, 1986 y otros más) se han ocupado de la problemática, del discurso comunitario como categoría social y de las relaciones entre lo público y lo privado en nuestras vidas. La comunidad moderna es mucho más compleja estructuralmente que la tradicional porque también lo es la sociedad en su conjunto y en todas sus manifestaciones sociales, culturales, deportivas, etc. Nada es tan sencillo como antes, nada es tan fácil en la modernidad como para entenderlo dentro de totalidades globales explicativas, que impregnen de sentido a nuestras decisiones personales. Caminamos por los 
viaductos de la especialización, sabiendo mucho de una cosa y casi nada de las demás. López Quintás (1982) apoda al mundo de la supraespecialización moderna como el "mal de Teresias", que nos obliga a profundizar en exceso en una dirección univoca y longitudinal, perdiendo la perspectiva de las globalidades humanas. Ya no percibimos a nuestro alrededor al hombre polifacético, al que sabe algo de todo, al humanista creativo o al erudito de todos los saberes. Estamos rodeados de especialistas por doquier. Nuestra sociedad busca lo concreto, lo que da rendimiento, lo que produce rápidos beneficios y casi siempre es eficaz. En el fondo, todo se valora en función del consumo que se convierte en la vitamina que revivifica las preocupaciones y hace públicas las carencias de la persona actual. Las funciones sociales de entrega, hospitalidad, compañerismo, amor altruista, colaboración desinteresada, etc., sufren la enfermedad del abandono, casi del olvido activo. Han quedado como símbolo y bandera de otras épocas más benévolas y participativas. El espacio público se fundamenta en relaciones espontáneas pero efímeras, en liturgias poco convincentes y en ritos protocolarios. Sin embargo no podemos renunciar a los beneficios que trae la convivencia humana participativa, a pesar del aislamiento $\mathrm{y}$ del individualismo que pregonan ciertas conductas sociales actuales urbanas (Habermas, 1981; Echeverría, 1995).

Sin duda fue Newbrough (1991) el que intentó definir y explicar la coyuntura histórica actual como el período postmoderno, en el cual los adelantos tecnológicos se incorporan a la vida social y se imponen como algo que nadie pone en duda. Esta nueva conquista humana (las tecnologías siempre son conquistas del hombre), trae consigo un nuevo planteamiento de la vida diaria de la población, donde el trabajo ya no es el destino exclusivo del hombre, sino que la cultura del tiempo libre y del ocio se van abriendo caminos y aparecen como nuevos espacios para la educación. Newbrough $(1991,1992)$ propone una nueva teoría de la comunidad que explique la complejidad de la vida social y la aparición de nuevos problemas como la marginación, la pobreza, la inadaptación social, la drogodependencia, la exclusión, el racismo, la xenofobia, etc., en el Estado del bienestar. Su teoría de la comunidad se conoce con el nombre de Sistema Social Humano. La idea principal de su teoría es la interdependencia entre los individuos organizados por vínculos escasamente rígidos. Los valores más destacados serían la ecología, el desarrollo, la interdependencia y el equilibrio. Estos valores propician que los individuos aprendan a participar usando sus propios recursos y así contribuyan al progreso de su propia comunidad. Los principios políticos que subyacen son la fraternidad, la libertad y la igualdad. La organización social de la comunidad debe arbitrar medidas de apoyo a todas las personas e instituciones y al mismo tiempo permitir que los individuos desarrollen soluciones personales. La moderna psicología comunitaria es deudora de esta teoría del Sistema Social Humano. Lo mismo podemos decir de las políticas sociales europeas que se basan en devolver la atención social a los grupos que la necesitan, principalmente la familia, los vecinos y las asociaciones voluntarias o las administrativamente reconocidas (asociaciones de vecinos, asociaciones de padres, etc.). 
El concepto moderno de comunidad está unido a otros como tolerancia, pluralismo, libertad, democracia, solidaridad, participación, interculturalismo, etc., dada la complejidad de la sociedad actual en todas las esferas de la vida convivencial (Illich, 1976; Fromm, 1978; Horney, 1979). La comunidad moderna se convierte en el lugar social de la acción. En ella se detectan personas, procesos, solidaridades, relaciones, conflictos, necesidades, recursos, instituciones, valores y normas. La comunidad, como microsociedad, plasma en su funcionamiento la realidad global con irrepetibles y diversas tonalidades. El Estado-nación como realidad del siglo xIx y como unidad política, ya no es el principio organizativo de todo el Estado moderno ni explica las distintas personalidades (con su propia cultura y lenguaje) de las regiones de un país. La ofensiva neoliberal ha tenido sus repercusiones en el campo social. Casi todo en Trabajo Social y en Servicios Sociales, además de las Ciencias de la Educación, se nos presenta bajo la etiqueta de comunidad. La comunidad no sólo tiene un gran poder simbólico, sino que carece de connotaciones negativas. El neoliberalismo recrea la ideología de la comunidad. El debilitamiento de los poderes de la comunidad nacional obligan a que cada persona busque con ahínco y esfuerzo las ayudas para su realización individual en las esferas de las comunidades locales. El hombre actual es consciente de que tanto el desarrollo nacional como la planificación del desarrollo global son ámbitos de actuación demasiado amplios y lejos de sus intereses y ocupaciones. Las gentes tienen la sensación de que dentro de las grandes organizaciones multinacionales no se puede hacer nada; los que ejercen el poder diseñan casi siempre los comportamientos colectivos de todos sus afiliados. De ahí que acuda a lo próximo, a lo local, para comprobar su participación efectiva y gratificante. Las comunidades del pasado (la polis griega, los municipios medievales, la democracia de las aldeas, las hacenderas) actuaban cara a cara y se comportaban como microcolectividades con unos mismos objetivos e intereses hacia la búsqueda de las conquistas sociales que beneficiaban a todos. Malagón (1998) hace un recorrido histórico interesante sobre los antecedentes remotos de la comunidad. Debemos recordar que la comunidad es un grupo social que se basa en el sentimiento que la gente tiene una de otra y cuyos miembros tienen conciencia de pertenencia e identificación con algún símbolo local. En la actualidad, estas microcolectividades se han convertido en macrocolectividades, el mundo moderno es el mundo de las corporaciones (Giner, 1983), de ahí la dificultad de regresar enfáticamente a esos mundos de convivencia de pequeño tamaño porque se ha creado un mundo donde las viejas comunidades son superfluas, ocupando su lugar las corporaciones por su capacidad para dar respuestas a los problemas que hoy se plantean.

Los procesos de industrialización están acompañados de una masiva huida de las personas y de las familias de los ámbitos rurales a los urbanos, creando graves problemas de asentamiento y una concentración masificada de trabajadores en barrios periféricos, donde las condiciones higiénico-sanitarias, sociales, educativas, etc., son las menos aconsejables para una calidad de vida normalizada. Este trasiego de personas que se lanzan a la búsqueda de trabajo fuera de sus contextos 
naturales (situación más grave la de los emigrantes a otros países europeos, donde la lengua es un enorme impedimento de relación comunicativa), nos obliga a reflexionar sobre una nueva fórmula de vivencia de la comunidad. La comunidad natural se convierte en un mundo nostálgico, porque generalmente nadie renuncia a sus raíces paternas y territoriales. La búsqueda de una comunidad segura se transforma en un anhelo y en una lucha en la que se involucran como una piña todos los miembros de la familia.

¿Cuáles son las reacciones que se producen en el seno familiar y en las personas en estas nuevas comunidades masificadas? La persona se encierra y únicamente comparte con los otros algunos momentos de su vida.

"La casa es un lugar donde el ser humano se refugia o simplemente se concentra con los suyos. Es un centro en el que se hacen converger los afanes y los caminos de la vida" (Rodríguez Neira, 1999: 95).

En realidad, no aparece la comunidad como sistema de convivencia, sino que aparecen distintas formas privadas grupales. Bellach (1989) ${ }^{4}$ lo explicita coherentemente:

"El estilo de vida es básicamente segmentario. Normalmente implica, de forma explícita, una oposición a otros que no comparten el estilo de vida de uno. Por eso, no hablamos de comunidades de estilos de vida, sino de enclaves de estilo de vida. Tales enclaves son segmentarios en dos sentidos. Sólo incluyen un segmento de cada individuo, ya que afectan únicamente a la vida privada, sobre todo al ocio y al consumo, y son socialmente segmentarios, ya que sólo implican a los individuos con un mismo estilo de vida".

La existencia del pluralismo y la segmentación de las tareas sociales nos obligan a cultivar la reciprocidad como conducta tolerante y a vivir juntos en la diferencia y con las diferencias. El interculturalismo y la pedagogía de la interculturalidad se imponen como metodologías activas para que la gente de todo el orbe se entiendan, se respeten y sean capaces de convivir conjuntamente. ¿Qué decir de las nuevas comunidades ciberespaciales y virtuales? Los espacios físicos y de contacto corporal han desaparecido. Todo se globaliza: la cultura, la economía, el interés generalizado por la moda o la canción que suena en todos los rincones. Como escribe Street (2000: 102):

"Uno de los problemas de las teorías de la globalización es su tendencia a ignorar las actividades de bajo nivel, lo que les impide percibir las relaciones que existen entre los aspectos locales, nacionales y globales".

4. Ballach, R. (1989): Hábitos del corazón. Alianza, Madrid, p. 105. 
Además del protagonismo del individuo en un mundo plural (las personas se desconocen aun viviendo en el mismo bloque de pisos), se exalta la vivencia del espacio privado y nada más íntimo y definidor de esa realidad social (la privacidad personal) que el propio hogar (vivienda y domicilio). El hogar se convierte en el refugio de la familia y en el aula de aprendizaje de todas las conductas sociales que se necesitan para sortear los peligros que aparecen fuera de él. Así lo expresa Schultz (1993) ${ }^{5}$ :

"El hogar es el punto de origen del sistema de coordenadas que aplicamos al mundo para orientarnos en él geográficamente, el hogar es determinado lugar de la superficie de la tierra. El lugar en que me encuentro, es mi morada; el lugar donde pienso permanecer, es mi residencia; el lugar de donde provengo y donde quiero retornar, es mi hogar".

Este retorno a lo privado como exigencia de los controles impuestos por la sociedad moderna justifica, de alguna manera, la pérdida de participación real en la vida de la comunidad, la aceptación pasiva de toda normativa (no conviene señalarse como discrepante) y la apariencia de que todos los individuos de la comunidad han llegado a ser ellos mismos. Desde este enfoque de la cultura narcisista,

"la defensa de la comunidad se erigirá sobre el mito de la solidaridad grupal, cuando ésta es más producto del miedo, la inseguridad y del temor a lo desconocido, que de las relaciones sociales" (Gurrutxaga, 1993) ${ }^{6}$.

Aparece como conducta social la figura del individuo que se protege de todo contagio exterior en el escenario de los espacios conquistados y que se va construyendo a sí mismo en medio de los ecoterritorios diversos mediante la simulación de los papeles sociales aprendidos. Los cambios sociales que se producen en la sociedad moderna (tecnológicos y productivos, crecimiento de las grandes ciudades, abandono de los ámbitos rurales, desarrollo de las tecnologías de la información y la comunicación, etc.) ejercen más efecto sobre los comportamientos de las personas que sobre los de la propia comunidad de pertenencia.

Muchas personas, incluso familias enteras, han abandonado la vida en la comunidad para emigrar a otras regiones o naciones con la finalidad de mejorar su nivel material de vida. En general, obtienen buenos resultados, pero deben pagar un alto precio social. En las comunidades rurales las gentes son tan escasas que se conocen bien unas a otras. Han nacido y crecido juntas. Viven y trabajan en el mismo territorio y confiesan que poseen un interés común que les anima a seguir habitando allí. Tienen y manifiestan un sentimiento de pertenencia. La emigración a las grandes urbes, por el contrario, hace que ese sentimiento de pertenencia se pierda rápidamente. La gente vive apartada, dedicada con exclusividad a su trabajo, si lo tiene.

5. Schultz, A. (1993): Estudios de teoría social. Amorrortu, Buenos Aires, p. 109.

6. GurrutXaga, A. (1993): "El sentido moderno de comunidad". Reis, 64, 211. 
3. LA ANIMACIÓN COMUNITARIA COMO ESPACIO ABIERTO INTEGRADOR Y TRASFORMADOR

Analizadas las características de la comunidad, sus cambios y problemas en la sociedad actual, nos resituamos en la animación comunitaria como un espacio abierto integrador y transformador en favor de los ciudadanos o colectividades para la consecución de la mejora del nivel de vida, mediante una conciencia y práctica social crítica (Sáez, 1998).

En nuestra literatura pedagógico-social aún se usa poco la terminología de animación comunitaria. Partimos de la base de que toda animación por definición es comunitaria, ya que tiene como elemento constructor el grupo social como microcomunidad. Lo mismo decimos de la educación social. Toda educación es social por naturaleza. Para Quintana (1993) 7 , la animación comunitaria es "la aplicación de técnicas propias de la animación a ciertos sectores del trabajo comunitario".

Cuando hablamos de animación comunitaria queremos ensalzar el aspecto comunitario de la animación, ya que sin él es imposible todo tipo de intervención social en la comunidad.

La animación comunitaria como ámbito de acción e intervención socioeducativa se centra en la comunidad y en todas sus circunstancias físicas, sociales, culturales, económicas, etc. Toda persona o grupo humano se desarrolla en una comunidad que como tal está constituida por las instituciones y las personas con las cuales nos relacionamos dentro de un territorio vital concreto. Quintana $(1993)^{8}$ escribe:

"La comunidad constituye, tanto para las personas como para los grupos, una importante unidad de vida y de relación. La comunidad complementa nuestra identidad personal dándonos una identidad social, y nos proporciona servicios y, con ellos, seguridad".

Entendemos la animación comunitaria (animación de la comunidad) como un proceso de acción socioeducativa que intenta, mediante las técnicas de la Animación, la sensibilización, la dinamización y la participación de todos los miembros de la comunidad en la transformación de su realidad global.

Examinemos con detalle la definición. Hablamos de un proceso de acción socioeducativa, lo que supone la existencia previsora de una planificación coherente, ordenada y secuencial de lo que se desea conseguir. De nada valen las acciones aisladas ni puntuales. La finalidad educativa es clara en toda acción interventiva con los miembros de la comunidad. Nos referimos a una educación integral, que abarque todos los aspectos de la persona (físicos, sociales, emocionales, comunicativos, mentales, etc.). Los éxitos de la comunidad se fundamentan en la preparación, el compromiso participativo y las actitudes colaborativas de cada uno de sus miembros. Esta faceta nunca debe olvidarse. Al contrario, es obligado potenciarla de una

7. Quintana, J. M. Los ámbitos profesionales de la Animación. Narcea, Madrid, p. 68.

8. Ibídem, p. 69 
forma continuada. Sólo así se incide sobre los órganos vitales de cualquier comunidad, que son las personas con sus identidades, su autoestima y su protagonismo. De ahí la importancia de la educación comunitaria como proceso educativo que da respuesta a las necesidades reales de las comunidades locales y busca todos los espacios posibles (escolares, deportivos, sociales, culturales, organizativos, etc.) para crear una mayor identificación de las personas con su comunidad, con la finalidad de mejorar su calidad de vida. Admitimos que los tres ejes fundamentales de la educación comunitaria son la comunidad, la participación y el aprendizaje. Pero este aprendizaje no se orienta exclusivamente a la escolarización; es un aprendizaje permanente, a lo largo de toda la vida (Delors, 1997). La educación comunitaria como realidad dinámica potencia un tipo específico de actividades educativas en función de los contextos socioculturales donde habita la población y según las necesidades de los individuos. Esta forma de entender la educación se opone a ese ritual místico donde los conocimientos se presentan como seguros y lejanos a los intereses de las personas, olvidando la contextualización de los aprendizajes. Ello, sin duda, explicaría el aburrimiento generalizado de este tipo de enseñanza.

El uso metodológico de las técnicas grupales de la animación es lo que da identidad propia a la animación comunitaria. Estas técnicas se pueden usar en cualquier tipo de actividades de carácter sociocomunitario: las fiestas populares, la protección de la cultura popular, la defensa del medio natural, el fomento del empleo juvenil, las visitas a los bienes culturales, el cultivo del deporte popular, los encuentros comarcales, la defensa de la salud de los ciudadanos, los proyectos de ocio y tiempo libre, las sesiones de participación ciudadana, los centros juveniles, las aulas de cultura, etc. La animación se convierte en un factor dinámico de las actividades de la comunidad. No basta con que las personas se yuxtapongan, es necesario que sean capaces de trabajar y crecer juntas. La animación estimula las relaciones humanas, suscita nuevas energías en los miembros de la comunidad, hace operativas las motivaciones personales e implica a las gentes en la búsqueda desinteresada del bien común.

4. La ANIMACIÓN COMUNITARIA COMO ESPACIO DE ACCIÓN SOCIOEDUCATIVA EN LAS COMUNIDADES LOCALES

Ante la enorme serie de problemas sociales de todo tipo (paro, pobreza, miseria, marginación, etc.), las comunidades locales o municipios adquieren importancia por su cercanía a la vida real de los ciudadanos. El tejido social se ha vuelto tan agresivo y tan anónimo que engendra escasa confianza en la propia historia personal y familiar. En la actualidad, se están potenciando las redes sociales (Barnes, 1954; Bott, 1990), como conjunto de relaciones que se establecen entre individuos y familias con su entorno más próximo. En las comunidades locales se pueden apreciar vínculos espontáneos, sentimientos de pertenencia e integración y deseos de colaboración desinteresada entre todos sus miembros. Luque (1995) escribe: 
"Por el contrario, en la sociedad actual prima el individualismo, la rivalidad y competencia entre sus miembros. Los ideales comunitarios tal y como tradicionalmente se han concebido chocan directamente con este modelo competitivo y corporativista que parece dominar en la sociedad actual.

La comunidad como tal es una construcción humana, donde el individuo se reconoce y donde el "nosotros" como realidad palpable identifica, de alguna forma, la individualidad con la existencia del grupo. De ahí que el compromiso personal expresado en el "nosotros" adquiera su máxima intensidad en aquellos grupos donde predominan los lazos afectivos: la familia y los amigos íntimos.

La preocupación por la temática de lo local es ya antigua, aunque su incorporación al campo científico sea relativamente reciente. La importancia de lo local en las políticas sociales hace que la población, objeto de la intervención, esté dispuesta a participar activamente en la búsqueda de soluciones de aquellas necesidades que invaden por doquier. Como aprecia Ander-Egg (1999) ${ }^{10}$ :

"Los programas de acción social comunitaria han ido ganando espacio en el ámbito de la sociedad civil, con el fin de transformar desde abajo la misma sociedad política y desarrollar nuevos modos de vivir".

Hablamos ahora de comunidad como sujeto de la intervención. En este sentido nos referimos a la asociación libre de personas dispuestas a desarrollar una acción socioeducativa ante las necesidades y problemas de su contexto más inmediato. Estas personas se saben parte del contexto en el que viven y se sienten afectados por él. Las necesidades constituyen la visualización del desorden existente en la red de sus relaciones y es necesario corregirlas cuanto antes.

Las políticas culturales municipales o locales son las que producen el beneficio participativo en las gentes. Las sociedades modernas anulan ciertas capacidades humanas necesarias para la puesta en marcha de proyectos comunitarios: la desconfianza en el otro, el engaño como símbolo de una mística profesional, el desprecio de las ideas y de las vidas ajenas, las incomunicaciones con los más cercanos y otro elenco de veleidades que dificultan las relaciones de comunicación entre las gentes. Para la creación de una política cultural local es necesario que las gentes que conforman esa comunidad sean capaces de trascender sus posiciones de apatía, pasividad o negatividad, hacia otras posturas más activas, más comprometidas y donde puedan reivindicar sus capacidades y su protagonismo en la vida comunitaria. A veces aparecen fenómenos de presión social (paros, huelgas, manifestaciones públicas, etc.) cuando se observa que las organizaciones institucionales no atienden a las necesidades de sectores concretos de la población civil.

9. Luque, P. A. (1995): Espacios educativos. Eub, Barcelona, p. 120.

10. Ander-Egg, E. (1999): ¿Qué es el desarrollo de la comunidad? Condinamarca, Bogotá, p. 144. 


\subsection{El compromiso de la comunidad}

Una de las obligaciones que impone la situación actual de nuestra sociedad, donde los recursos son más bien escasos y las necesidades/problemas abundantes, es la recuperación de los aspectos preventivos de las necesidades sociales o la intervención educativa que implica tener conciencia de la necesidad de incidir sobre el proceso educativo. Es una lucha en la que todas las fuerzas sociales y políticas están comprometidas, conjuntamente. Se convierte en axioma operativo a nivel programático el deseo profesional de minimizar las necesidades sociales (generalmente numerosas) y maximizar los recursos existentes (normalmente escasos). ¿Es todo esto posible, socialmente? Estamos de acuerdo con Marchioni (1994) ${ }^{11}$ que la prevención verdadera sólo es posible a niveles colectivos, ya que modifica valores, actitudes, sentimientos y comportamientos que se producen en la colectividad. Rodríguez Neira (1996: 15) apunta en la misma línea: "No tiene sentido intervenir en las partes aisladamente. Sólo es posible actuar sobre una unidad teniendo en cuenta al conjunto".

La prevención debe llevar a un cambio de los valores y de los comportamientos, aunque sea de una forma lenta pero ideológica. Los cambios ideológicos u axiológicos siempre languidecen por su perennidad, pero, al final, consiguen sus propósitos. Es cuestión de creer en las utopías posibles.

Prevenir en la comunidad significa un estudio pormenorizado y global de la etiología de los problemas/necesidades que padece el colectivo. Sin la lucha por la eliminación de las posibles causas que determinan los problemas, jamás se producirán cambios colectivos e individuales. Incidir sobre las causas que producen los problemas nos obliga a una planificación racional, coherente y responsable y a una coordinación conjunta, ya que las causas nunca aparecen como unilaterales. Esta forma de entender las actuaciones pedagógico-sociales es bautizada por Colom (1985: 41) como intervención pasiva, en cuanto implica la preparación y programación de la acción, previa a la acción misma.

Se impone una valoración de lo local, de lo cercano a la vida de las personas y de las colectividades. Sólo así conseguiremos que la animación como sistema de comunicación sea un instrumento válido para la revitalización del tejido social. La metodología de la animación comunitaria nos obliga a concretar la identidad cultural territorial como motor que moviliza el proceso de animación y que permite a los colectivos asumir el propio entorno sociocultural. Asumir la identidad cultural como algo que nos pertenece y que nos empuja a la acción inevitablemente es uno de los factores prioritarios a la hora de diseñar proyectos de intervención sociocultural. La gestación cultural territorial se convierte en un paradigma comunitario. Siempre actuamos/intervenimos sobre territorios concretos, definidos por una serie de coordenadas que aparecen como el reflejo más coherente de su vida cotidiana

11. Marchioni, M. (1994): La utopia posible. Benchomo, Tenerife, pp. 89-90. 
bajo la práctica de la participación y la solidaridad social. Es obligado que en tales circunstancias ambientales y psicosociales, el animador establezca una serie de normas o de principios generales que faciliten o favorezcan la participación de la gente. Además de la señalización de los objetivos concretos a conseguir, se debe intentar que las propuestas participativas obtengan algún beneficio inmediato y tangible, de tal modo que la recompensa funcione como el motor que da fuerzas y ánimos para seguir adelante cuando los procesos sean largos y difíciles. De lo contrario, el desánimo y el desasosiego afectivo sacude todas las actuaciones de la persona o pone en peligro la continuidad del proyecto de intervención.

Toda acción sociocomunitaria en los ámbitos locales (centros cívicos, asociaciones, concejalías, municipios, etc.) implica el respeto a la pluralidad de las manifestaciones culturales que se puedan producir. Como escribe Ander-Egg $(1997)^{12}$ :

"Las ha de promover y potenciar cualquiera sea su opción política particular, siempre que la potenciación de esas actividades culturales sirva para consolidar la convivencia democrática y permita el desarrollo de individuos, grupos y comunidades".

Las acciones culturales se deben descentralizar y realizarlas en los lugares donde la gente habite: barrios, suburbios, comunidades de base, aldeas, pueblos, mercados, asociaciones, cooperativas, talleres protegidos, etc.

Las comunidades locales serán cada día más protagonistas de su propio desarrollo. Desarrollo integral que afecta a todos los sectores de la población y a todas sus manifestaciones: económicas, sociales, culturales, políticas, ambientales, sanitarias, etc. Las actitudes solidarias aparecen en los distintos espacios, siendo el más importante la institución familiar, a pesar de su crisis actual. No podemos olvidar que el proceso de socialización cubre una de sus fundamentales etapas evolutivas en el mundo comunitario (socialización primaria y secundaria). Dentro de la dinámica de la sociedad actual se impone como una necesidad imperiosa, y por la que debemos luchar sin denuedo, la recuperación de los espacios locales (vecindades, comunidades, barrios, pueblos, etc.). Ahí, en esos ambientes convivenciales es donde se puede crear una conciencia social transitiva que implique a las gentes en acciones transformadoras. Como apostrofa Kisnerman $(1986)^{13}$ :

"Un proceso de estimulación a partir del cual los pobladores de un espacio se transforman en vecinos, que como tal toman conciencia de sus problemas colectivos, conozcan sus recursos, aptitudes y capacidades para afrontar esos problemas, elaboren un plan de acción y logren la comunidad que desean".

El desarrollo de las capacidades sociales (sentimiento comunitario) se produce mediante un proceso de tres fases: conciencia social, organización social y transformación social. En la puesta en marcha de este proceso es donde adquieren su

12. Ander-Egg, E. (1997): Metodologías de Acción Social. ICsA, Buenos Aires, p. 60.

13. Kisnerman, N. (1986): Comunidad. Humanitas, Buenos Aires, p. 68. 
verdadero papel las redes sociales. En todo contexto social inmediato existen una serie de cauces de relación social, más o menos informales, que constituyen el entramado que sustenta las aspiraciones de mejora de cada colectivo humano. Debemos ponerlas al servicio de la propia comunidad, en cuanto manifiestan la atmósfera emotiva de la persona en la vida de la comunidad.

\subsection{Cohesión social e inserción laboral}

Actuar con la comunidad exige para su éxito que los distintos estamentos que la componen: Administración, técnicos, profesionales y ciudadanos deben cooperar solidariamente y plantearse objetivos comunes. Implica la armonía comprometida de todo el tejido social en las intervenciones comunitarias con la finalidad de evitar o neutralizar las discriminaciones racistas o sexistas y luchar contra la exclusión y la marginación social. Ello supone que cada colectivo debe desempeñar su papel y aportar lo mejor en beneficio de la comunidad, porque el proyecto comunitario comporta un compromiso firme con la educación por parte de los diversos agentes sociales y culturales. Por desgracia, no siempre ha sido así. Parece que dos mundos diferenciados y casi enfrentados luchaban por el bien común: por un lado las administraciones y, por otro, los ciudadanos como simples usuarios. De ahí que el modelo de intervención deba orientarse de nuevo y no sufrir los esquemas convencionales de reducir los clientes a simples usuarios. Es necesario responsabilizar a la población de la gestión de sus propias necesidades. Con frecuencia, cuando se habla de comunidad parece que uno se refiere con exclusividad a los ciudadanos, pero como comenta Marchioni (1994) ${ }^{14}$ :

"Los protagonistas reales de cualquier proceso participativo en un sistema democrático son tres: la Administración, los servicios y profesionales públicos y la población”.

Es importante subrayar la dimensión comunitaria de la intervención. Como comenta León $(1995)^{15}$ :

"Las acciones desarrolladas en los distintos niveles de atención (individual, grupal e institucional) han de interrelacionarse entre ellas y con el contexto comunitario en el que conviven las personas, grupos y organizaciones. Señalar el objetivo de trabajar con la comunidad y diseminar el saber profesional en procesos de ayuda y capacitación a los ciudadanos, permite al interventor experimentar saludables procesos de desprofesionalización y a la comunidad no mantener relaciones de dependencia con los técnicos".

14. Ibídem, p. 125

15. LEÓN, J C. (1995): "La psicología comunitaria: una perspectiva en la intervención social". En Conde, J. e Isidro, A. I.: Psicología Comunitaria, Salud y Calidad de Vida. Eudema, Madrid, p. 47. 
Los procesos de sensibilización y dinamización de la comunidad son pasos previos para la consecución de la participación activa de los miembros de esa comunidad. La participación comunitaria, como toda participación, se hace con el propósito de alcanzar determinados objetivos, satisfacer necesidades, resolver problemas o desempeñar funciones sociales relevantes a nivel local. Para Pérez Serrano $(1993)^{16}$ la sensibilización se lleva a cabo mediante tres etapas:

\subsubsection{Concienciación/conciencia transitiva}

Implica despertar el interés de las personas por algo que tenga significado para sus vidas. Supone un conocimiento amplio de sus centros de interés, de sus disponibilidades y de sus ganas de actuación en beneficio propio y de los demás. Se trata de crear en los miembros de la comunidad la necesidad de estar correctamente informados, de conocer en profundidad su propia realidad (posturas, intereses, conflictos, etc.). Como escribe Vilar (1996) ${ }^{17}$ :

"Es un trabajo dirigido al autoconocimiento del grupo al que se dirige el proyecto, consistente en que las personas que deban ser sujetos activos de la intervención sean conscientes de su realidad, de los aspectos autobiográficos que los han llevado a su situación actual, de sus posibilidades reales y del momento en el que se encuentran, lo que debe despertar el interés por el trabajo que se les propone realizar".

\subsubsection{Información/formación/promoción}

Se deben arbitrar todas las medidas oportunas para que los ciudadanos conozcan su entorno y sean capaces de tomar una postura ante él. La persona bien informada es la más libre a la hora de la toma de decisiones. Los ciudadanos desean conocer los hechos y los datos, los problemas y las posibles soluciones para comprometerse personalmente. A veces, es necesario formar a la población, dada su desventaja sociocultural en todas las facetas instructivas del conocimiento. La formación (la Bildung alemana) la entendemos como toda intervención que produce cambios en el comportamiento, en los conocimientos, en la información y en las aptitudes y que no se deben al desarrollo natural de la persona.

"La información debe ser asumida como una constante del proceso y como parte del trabajo del equipo comunitario en particular" (Marchioni, 1999) ${ }^{18}$.

16. Pérez Serrano, G. (1993): Elaboración de proyectos sociales. Casos prácticos. Narcea, Madrid, p. 105.

17. VILAR, J. (1996): «De la planificación a la programación”. Educación Social, 3, 42.

18. Marchioni, M. (1999): Comunidad, participación y desarrollo. Popular, Madrid, p. 105. 
Este criterio de información/formación debe ir encaminado hacia la promoción y la inserción social y laboral. El nivel informativo/formativo exige la puesta en práctica de todas aquellas ofertas de formación continua con el objetivo de capacitar a las personas en el aprendizaje y gestión del conocimiento en los ámbitos científico, cultural y tecnológico. Todas las actividades actúan como acciones de promoción dentro de un largo proceso de adquisición de nuevas pautas de comportamiento.

\subsubsection{Interpretación de la historia y la cultura}

Se pretende que cada miembro se identifique con la realidad presente de su comunidad y sea capaz de interpretar las demás estructuras (sociales, económicas, culturales, políticas, sanitarias, medioambientales, etc.) que la componen.

Esta fase básica de sensibilización nos permitirá abordar con éxito el proceso sistémico para la elaboración de los proyectos comunitarios de intervención, puesto que toda la comunidad, especialmente los estamentos más activos, deben interpretar correctamente todos los elementos del diseño de cara a los objetivos. Para ello debemos plantear un proceso de sensibilización, de tal manera que se vayan implicando progresivamente todos los miembros de la comunidad en la medida de sus posibilidades reales y muestren su grado de implicación en el desarrollo y ejecución del proyecto comunitario. El objetivo fundamental de la sensibilización es lograr que toda la comunidad reconozca su interés en el proyecto comunitario y se implique de alguna manera en el proceso de su elaboración y ejecución (puesta en práctica). El animador comunitario iniciará el proceso, presentando a la comunidad los motivos de la puesta en marcha de un proyecto de intervención social, informando sobre todos aquellos aspectos que la gente requiera y promoviendo el primer debate/coloquio. Se necesita llegar a un acuerdo básico sobre la función del proyecto, base del interés de la comunidad para su ejecución. Posteriormente, se presentará una propuesta de actuación, se someterá a debate y se garantizará el nivel participativo de la comunidad, recogiendo sus aportaciones y sugerencias en reuniones colectivas.

Superada la fase de sensibilización de la comunidad (que puede durar bastante tiempo; no es aconsejable precipitarse, hasta que la gente no se identifique con su realidad social problemática) se pasará a la búsqueda de proyectos alternativos propios, nacidos de la dinamización social de la comunidad. Pérez Serrano $(1993)^{19}$ escribe:

"La dinamización ofrece una reorientación del camino recorrido, una revisión de los planteamientos anteriores, una readaptación a perspectivas de progreso colectivo".

19. Ibídem, p. 108. 
Nada es mejor que la misma comunidad se comprometa a buscar soluciones a sus problemas o necesidades. Es lo que Colom (1985: 41) bautiza como intervención activa que se centra en la definición de objetivos, en la acción y en el proceso evaluador. Ello implica la elaboración de un diagnóstico contextual de la problemática (recogida de todo aquello que es relevante dentro del seno de la propia comunidad), la propuesta de posibles soluciones u objetivos a conseguir, las actividades, la metodología, el cronograma y la búsqueda de recursos de toda índole, antes de realizar la evaluación de resultados finales. La retroalimentación nos irá señalando las metas conseguidas o los fallos que deben ser corregidos, puntualmente.

Elaborado comunitariamente el diseño del proyecto de intervención social (donde cada grupo tendrá su tarea para desempeñar/ejercer), estamos en condiciones óptimas de que la gente participe activamente en su realización/ejecución. Como comenta Merino $(1997)^{20}$ :

"Las tareas de información, implicación y formación de los destinatarios constituyen una de las actividades claves en el proceso de planificación y ejecución de los programas".

\subsection{El nivel programador de las intervenciones comunitarias}

Elegida una metodología de intervención social por la comunidad, ella misma debe seguir el proceso de actuación, un proceso retroactivo que supone el estudio de aquellos aspectos de la realidad sobre los que deseamos actuar o incidir. Entramos de lleno en el nivel programador de las acciones comunitarias. El cruce entre el análisis concreto de necesidades y la valoración de los recursos disponibles determinará las tácticas del programa. La intervención comunitaria requiere la metodología planificadora/programadora como una estrategia permanente y normalizada. De lo contrario, es imposible el proceso comunitario. Se trata de tener información abundante sobre las necesidades, los problemas, los recursos, las potencialidades y los niveles de compromiso participativo, etc., y ello se nos presenta como el diagnóstico de la situación. Es el momento del análisis de la propia realidad social. Tenemos ya el diagnóstico contextual. Conocemos con detalle toda la problemática social, económica, cultural, sanitaria, educativa, etc., de la comunidad. El estudio de campo nos ha proporcionado una serie de datos que debemos valorar e interpretar. Todo ello nos obliga a una jerarquización de las necesidades y sus posibles soluciones. Este análisis completo de la realidad social supone la existencia de tres niveles circulares de explicación, para una posterior acción transformadora. Estos tres niveles son: nivel de observación/descripción, nivel crítico/descodificado y nivel creativo o transformador (Froufe, 1996) ${ }^{21}$.

20. Merino, J. V. (1997): Programas de Animación Sociocultural. Narcea, Madrid, p. 187.

21. Froljfe, S. (1996): Planificación e Intervención Socioeducativa. Amarú Ediciones, Salamanca, pp. 114-116. 
El diagnóstico comunitario significa «la necesidad y la voluntad de compartir entre todos los recursos, una visión global e integrada de la comunidad en la que todos intervienen, aunque cada uno en su ámbito y con sus competencias» (Marchioni, 1999) ${ }^{22}$. Después diseñaremos los objetivos que deseamos conseguir y programaremos aquellas actividades necesarias para producir el cambio de la situación presente/real a una deseada. Anticipadamente, establecemos el camino (ámbito metodológico) que debemos seguir para conseguir los objetivos deseables y propuestos de antemano. Seguidamente vendrá la fase más importante: la ejecución real del proyecto. Se planifica y proyecta para ponerla en práctica. El sentido pedagógico de la planificación social se encuentra en su proyección dinámica para producir cambios sociales y reducir las necesidades de la población.

"Implica llevar a cabo un conjunto de acciones que se realizan dentro de un determinado ámbito, condicionado por contextos más amplios y que dan lugar a un juego incierto de interretroacciones" (Ander-Egg, 1997) ${ }^{23}$.

Finalmente, vendría la etapa evaluativa como forma de valorar la ejecución o puesta en práctica de la intervención social (la evaluación como tal abarca todas las fases: diagnóstico, programación y ejecución). La evaluación entendida así figura como un instrumento que posibilita el proceso de planificación sociocomunitaria como un proceso vivo y dinámico, en constante retroalimentación para adaptarse de una forma mejor y más provechosa a las necesidades que intenta eliminar o reducir.

Toda intervención comunitaria en las nuevas condiciones sociales parte siempre de los objetivos que se proponga la misma comunidad. No se pueden esperar soluciones de los ajenos/extraños a sus propias vivencias. Tal vez sea obligado sensibilizarlos, el que tomen parte activa en los programas comunitarios y pongan empeño en que las acciones comunitarias sigan un camino realista y práctico con la finalidad de mejorar los niveles de vida de todos los ciudadanos.

\section{CONCLUSIONES}

Esbozamos, en forma de sintesis, aquellos principios normativos que son imprescindibles a la hora de una acción e intervención comunitaria integradora como un espacio abierto a la participación de todo el colectivo social. Figuran como simples propuestas que se deben llevar a cabo en beneficio de la comunidad y sus miembros y que aparecen flexibles a cualquier tipo de sugerencia.

a. La moderna vivencia de la comunidad actual poco tiene que ver con la tradicional. Ciertos valores sociales han desaparecido. Han nacido otros distintos. El

22. Ibídem, p. 123.

23. Ibídem, p. 56 
hombre necesita ser fiel a su propia historia social y personal. El moderno concepto de comunidad va unido a pluralismo, interculturalismo, democracia, libertad, tolerancia, etc., aunque aparezcan, de vez en cuando, ciertos atisbos de xenofobia, racismo y otras singladuras escasamente justas (paro, marginación, pobreza, etc.). La humanidad de las conductas humanas aparece por doquier.

b. Importancia de la educación comunitaria como proceso educativo que intenta dar respuesta a los problemas reales de las comunidades desde todos los ámbitos posibles (culturales, deportivos, sanitarios, ecológicos, organizativos, etc.). La educación como práctica de la libertad personal y de los pueblos (Freire, 1986).

c. La acción e intervención comunitaria integradora presupone que todos los elementos orgánicos deben coordinarse para la consecución de fines comunes. Administración, servicios profesionales y ciudadanos son la razón de ser de los servicios públicos. Los colectivos sociales además de ser usuarios de los proyectos de intervención social, son ciudadanos con todo el engranaje de problemas que esto supone en una sociedad tan abierta e insolidaria como la nuestra. Como escribe Marchioni (1989) ${ }^{24}$ :

"No es suficiente luchar por objetivos justos; hay que hacerlo, además, con métodos correctos. Para ello es necesario analizar críticamente el pasado, reconocer y corregir los errores, avanzar hacia el futuro desde un presente zarandeado por muchos y muy profundos cambios".

d. Es necesario recuperar la idea de lo colectivo, de lo grupal, de lo comunitario, donde se supere la visión de lo personal/lo privado, aunque siempre se tenga en cuenta a la hora de las acciones e intervenciones sociales. Las causas de los problemas son siempre generales. Las acciones e intervenciones socioeducativas deben ser globales en todas las fases del diseño, sin olvidar las problemáticas y las virtualidades personales.

e. Es urgente recuperar el sentido de la verdadera prevención social. Debemos incidir en la modificación de actitudes, valores y comportamientos colectivos. La verdadera prevención llegará al conocimiento de los factores multicausales de riesgo de tipo macro y microsocial y de todas sus influencias en posteriores comportamientos sociales. Es necesario usar todos los espacios comunitarios abiertos en la intervención educativa

"que defenderá la acción voluntarista de participación activa a la hora de aportar un programa educativo, con lo que el proceso pedagógico se orientará, forzosamente, hacia metas u objetivos" (Colom, 1985: 40).

24. MARChIONI, M. (1989): Planificación social y organización de la comunidad. Popular, Madrid, p. 83 . 
f. El modelo de intervención que defendemos supone una estrategia de carácter interdisciplinario e integrador, donde los esfuerzos y los niveles participativos de la comunidad van a ser el cimiento del posible éxito de la realización del proyecto de animación comunitaria. Es necesario trabajar con/en/para la comunidad como si se tratara de un equipo multidisciplinar y multiprofesional, donde cada agente cumple con unas funciones específicas. Los modelos comunitarios mediante las redes sociales se imponen en la actualidad en casi todos los ámbitos de la intervención socioeducativa, sin olvidar nunca a la persona como ser individual, con sus valores, actitudes, cualidades y también defectos.

g. La cultura popular debe considerarse como una forma de autoexpresión de las vivencias de los ciudadanos. La utilización de la cultura popular o la identidad cultural territorial como afirmación de la autonomía de los pueblos es la mejor vitamina para luchar contra todo tipo de opresión política indiscriminada. La cultura popular se convierte así en una "cultura de la resistencia", en un instrumento de cambio político y en un motor de reformas a favor de los ciudadanos y de todos sus espacios sociales: familia, grupos primarios, vecindad, comunidad, hábitat, ambiente, Administración pública, etc.

\section{BiBLIOGRAFÍA}

Ander-EgG, E. (1997): Metodologias de Acción Social. ICSA, Buenos Aires.

- (1999): ¿Qué es el desarrollo de la comunidad? Condinamarca, Bogotá.

ARIES, Ph. y Duby, G. (1989): Historia de la vida privada. Taurus, Madrid.

BASSAND, M. (1992): Cultura y Regiones de Europa. Oikos-Tau, Barcelona.

BELlACH, R. (1989): Hábitos del corazón. Alianza, Madrid.

BotT, A. (1990): Familia y red social. Taurus, Madrid.

Bunge, M. (1985): Seudociencia e ideologia. Alianza, Madrid.

CARBONELL, J. (2001): La aventura de innovar. Morata, Madrid.

CASTELLS, M. (1997): La era de la información. Economia, sociología y cultura. La sociedad red. Alianza, Madrid.

Colom, A. J. (1985): "Educación y Municipios”. En AA.VV. (1985): Condicionamientos sociopolíticos de la educación. CEAC, Barcelona, pp. 35-54.

- et al. (1987): Modelos de Intervención Socioeducativa. Narcea, Madrid.

Cortina, A. (1997): Ciudadanos del mundo. Alianza, Madrid.

Delgado, M. (1999): El animal público. Hacia una antropología de los espacios urbanos. Anagrama, Barcelona.

DeLors, J. (1997): La educación encierra un tesoro. Santillana, Madrid.

EcheVerría, J. (1995): Cosmopolitas domésticos. Anagrama, Barcelona.

Fermoso, P. (1994): Pedagogía social. Fundamentación científica. Herder, Barcelona.

FreIRE, P. (1986): La educación como práctica de la libertad. Siglo xxI, Barcelona.

Froufe, S. (1996): Planificación e Intervención Socioeducativa. Amarú Ediciones, Salamanca.

- (1998): Las Técnicas de grupo en la Animación Comunitaria. Amarú Ediciones, Salamanca.

- (1999): "Acercamento conceptual ao Estatuto Epistemolóxico da Animação Comunitaria". Anasc (Portugal), 4, 51-60. 
Gergen, K. G. (1992): El yo saturado. Paidós, Barcelona.

GINER, S. (1983): Comunidades sociales adultas. Mezquita, Madrid.

GuRRUTXAGA, A. (1993): «El sentido moderno de la comunidad”. Reis, 64, 201-219.

HaBermas, J. (1981): Historia y crítica de la opinión pública. Gustavo Gili, Barcelona.

KisNeRMAN, N. (1986): Comunidad. Humanitas, Buenos Aires.

LEÓN, J. C. (1995): "La psicología social comunitaria: una perspectiva en la intervención social.. En Conde, J. e Isidro, A. I. Psicología Comunitaria, Salud y Calidad de Vida. Eudema, Madrid, pp. 39-51.

Lipovetsky, G. (1990): La era del vacío. Ensayos sobre el individualismo contemporáneo. Anagrama, Barcelona.

LUQUE, P. A. (1995): Espacios educativos. Sobre la participación y transformación social. eUB. Barcelona.

Malagón, J. L. (1998): Fundamentos del Trabajo Social Comunitario. Universidad Pablo de Olavide, Sevilla.

Marchioni, M. (1989): Planificación social y organización de la comunidad. Popular, Madrid.

- (1994): La utopia posible. Benchomo, Tenerife.

- (1999): Comunidad, participación y desarrollo. Teoría y metodología de la intervención comunitaria. Popular, Madrid.

MeRINo, J. V. (1997): Programas de animación sociocultural. Narcea, Madrid.

Nassir, R. (1980): Teoría de la Educación. Cincel-Kapelusz, Buenos Aires.

Newbrough, J. R. (1991): "Hacia una teoría de la comunidad para la Psicología Comunitaria”. Revista Interamericana de Psicologia, 25, 1-22.

Pérez Serrano, G. (1993): Elaboración de proyectos sociales. Casos prácticos. Narcea, Madrid. Petrus, A. (1997): Pedagogía social. Ariel, Barcelona.

POPKEWITZ, T. S. (1988): Paradigma e ideología en investigación educativa. Mondadori, Madrid.

Porzecansky, T. (1989): Desarrollo de comunidades y subculturas. Humanitas, Buenos Aires.

Postman, N. (1994): Tecnópolis. La rendición de la cultura a la tecnología. Círculo de Lectores, Barcelona.

RIFKIN, J. (1996): El fin del trabajo. Paidós, Barcelona.

Roвotrom, I. M. (1987): Environmental Education: Practice and Possibility. Deakin University Press, Geelong.

Rodríguez Neira, T. (1996): "Aprendizajes tácitos: comunidades, grupos y casas". Aula abierta, 68, 3-18.

- (1999): "Lo privado. Aprendizajes tácitos". Teoría de la Educación. Revista Interuniversitaria, 11, 85-100.

Quintana, J. M. (1993): Los ámbitos profesionales de la Animación. Narcea, Madrid.

Schultz, A. (1993): Estudios de teoria social. Amorrortu, Buenos Aires.

StReEt, J. (2000): Política y cultura popular. Alianza, Madrid.

VICHÉ, M. (1999): Una pedagogía de la cultura: la animación sociocultural. Certeza, Zaragoza.

VILAR, J. (1996): "De la planificación a la programación”. Educación Social, 3, 11-49. 